\title{
Das Bürzeldrüsensekret der Reiherente (Aythya fuligula)
}

The Uropygial Gland Wax from the Tufted Duck

\author{
JÜRGEN JACOB * \\ Universität Hamburg \\ und \\ Alfred Zeman **
}

Unilever-Forschungslaboratorium Hamburg

(Z. Naturforsch. 25 b, 1438-1447 [1970] ; eingegangen am 31. Juli 1970)

\begin{abstract}
The acidic and alcoholic components of the uropygial gland wax from the tufted duck (Aythya fuligula) were investigated by a combination of GLC and mass spectrometry. Main components of the acidic part of the waxes are 2,4,6-trimethyl-nonanoic $(39.0 \%) 2,4,6$-trimethyl-octanoic $(8.7 \%)$ and 2,6-dimethyl nonanoic acid (5.9\%). Besides, 2,4,8-, 2,6,8-, and 2,6,10-trimethyl-substituted fatty acids occur. As minor components 2-methyl- and homologous series of 2,4-, 2,6-, 2,8-, and 2,10-dimethyl-substituted fatty acids could be detected.

Main components of the wax alcohols were n-hexadecanol and n-octadecanol. As minor components 2-, 6-, 10-, 12-, and 14-methyl-branched primary alkanols occur.

The tufted duck shows an uropygial gland wax which is typical for the 17th order of the natural system.
\end{abstract}

Zahlreiche Untersuchungen der letzten Jahre über die Bürzeldrüsensekrete verschiedener Vögel haben einen Zusammenhang zwischen der chemischen $\mathrm{Zu}$ sammensetzung dieses Sekretes und ihrer Zugehörigkeit zu bestimmten Ordnungen des natürlichen Systems erkennen lassen. So ist das Vorkommen von $n$-Alkan-2.3-diolen für die Arten der Hühnervögel (18. Ordnung, Galliformes) charakteristisch ${ }^{1-4}$, während bei den Gänseartigen (17. Ordnung, Anseres) das Auftreten polymethylsubstituierter Fettsäuren chemotaxonomisches Merkmal ist $^{5-16}$. Über die heterogene Vogelgruppe der 20. Ordnung (Laro-limicolae) liegen erst 2 Publikationen vor ${ }^{17}$, 18 , die bislang keine chemotaxono-

Sonderdruckanforderungen an Dr. rer. nat. JÜRGEN JACOB, D-2000 Hamburg-66, Kakenhaner Weg 14.

* Anschrift des Verfassers: Dr. Jürgen JaCob, 2000 Ham. burg-66, Kakenhaner Weg 14. ** Dr. Alfred Zeman, Unilever-Forschungslaboratorium Hamburg, 2000 Hamburg-50, Behringstr. 154.

1 I. A. Hansen, B. K. Tang u. E. Edrins, J. Lipid Res. 10, 267 [1969].

2 E. O. A. HaAthi u. H. M. Fales, J. Lipid Res. 8, 131 [1967].

3 J. Jaсов u. G. Grimmer, Z. Naturforsch. 25 b, 577 [1970].

4 J. Jасов u. G. Grimmer, Z. Naturforsch. 25 b, 689 [1970].

5 G. Weitzel u. K. Lennert, Hoppe-Seyler's Z. physiol. Chem. 288, 251 [1951].

${ }^{6}$ K. Lennert u. G. Weitzel, Hoppe-Seyler's Z. physiol. Chem. 288, 266 [1951]. mischen Schlüsse zulassen; dagegen scheinen in der 32. Ordnung (Passeres) 2-methyl- und vor allem 3methylsubstituierte Fettsäuren als Bestandteile des Bürzelwachses zu dominieren ${ }^{19-21}$.

Die vornehmlich von ODHAM untersuchte 17. Ordnung ist in fast allen Unterfamilien mit einem näher analysierten Exemplar belegt. Es fehlen in der Unterfamilie Anatinae lediglich Untersuchungen der Tribus Aythyini und Oxyurini. Aus der ersteren stand uns jetzt ein Exemplar in der Reiherente (Aythya fuligula) zur Verfügung, deren Bürzeldrüsensekret in der vorliegenden Arbeit analysiert wurde.

7 G. Weitzel, A.-M. Fretzdorff u. J. Wojahn, Hoppe-Seyler's Z. physiol. Chem. 291, 29 [1952].

8 G. Weitzel, A.-M. Fretzdorff u. J. Wojahn, Hoppe-Seyler's Z. physiol. Chem. 291, 46 [1952].

9 G. Odham, Ark. Kemi 21, 379 [1963].

10 G. Odham, Ark. Kemi 22, 417 [1964].

11 G. OdHam, Ark. Kemi 23, 431 [1965].

12 G. Odham, Ark. Kemi 25, 543 [1966].

13 G. ODham, Ark. Kemi 27, 251 [1967].

14 G. Odham, Ark. Kemi 27, 263 [1967].

15 G. ODHam, Ark. Kemi 27, 289 [1967].

16 G. OdHam, Ark. Kemi 27, 295 [1967].

17 H. Karlsson u. G. Odham, Ark. Kemi 31, 143 [1969].

18 J. Jасов u. G. Grimmer, Z. Naturforsch. 25 b, 54 [1970].

19 J. JACOB U. A. Glaser, Z. Naturforsch., im Druck.

20 J. JACOB u. A. Zeman, Z. Naturforsch. 25 b, 984 [1970].

21 J. JAсов u. A. Zeman, Z. Naturforsch., in Vorbereitung. 


\section{Material und Methode}

\section{Material}

Die Bürzeldrüse einer frisch getöteten weiblichen Reiherente (Behrensdorf, Hohwachter Bucht) wurde präpariert und in der üblichen Weise aufgearbeitet ${ }^{19}$.

\section{Aufarbeitung}

Die Extraktion mit $\mathrm{CHCl}_{3} / \mathrm{CH}_{3} \mathrm{OH}(2: 1)$ lieferte nach Trennung der Phase durch Zugabe von Wasser die Rohlipide $(173,1 \mathrm{mg})$. Ein Teil davon $(132 \mathrm{mg})$ wurde über eine 5 -g- $\mathrm{SiO}_{2}$-Säule durch Elution mit $\mathrm{Cy}$ clohexan $+15 \%$ Benzol (100 ml) gereinigt. Die resultierende Fraktion $(127,6 \mathrm{mg})$ erwies sich dünnschichtchromatographisch als einheitlich. Die Umesterung mit 5-proz. methanolischer $\mathrm{HCl}$ und anschließende säulenchromatographische Trennung ${ }^{19}$ ergab die Methylester $(61,2 \mathrm{mg})$ und Alkohole $(65,6 \mathrm{mg})$. Nach GLC-Untersuchungen wurden die Alkohole $(60,1 \mathrm{mg})$ mit $30 \mathrm{ml}$ des Chromsäure-Oxydationsgemisches ${ }^{20}$ versetzt und die resultierenden Fettsäuren in der üblichen Weise aufgearbeitet.

\section{Gaschromatographie (GLC)}

Die GLC wurde bereits früher ausführlich beschrieben ${ }^{18-20}$.

\section{Massenspektrometrie (MS)}

Für die GLC-MS-Kombination wurde eine 50-m-Kapillarsäure mit SE 30 als stationäre Phase (PerkinEImer Typ 11G20) benutzt, die über einen einstufigen Biemann-Separator an ein einfach-fokussierendes Massenspektrometer (Varian CH 7) angekoppelt war.

Die GLC der Wachs-fettsäuremethylester wurde bei programmierter Säulentemperatur $\left(100-200^{\circ}\right.$ mit $10^{\circ}$ Min., Injektionstemperatur $300^{\circ}$, Separatortemperatur $250^{\circ}$, Trägergasstrom $2 \mathrm{ml} \mathrm{He} / \mathrm{Min}$.) ausgeführt.

Die GLC der aus den Alkoholen resultierenden Methylester wurde ebenfalls im Temperaturprogramm von $200-250^{\circ}$ mit $10^{\circ} / \mathrm{Min}$. unter sonst gleichen Bedingungen vorgenommen. Die Registrierung erfolgte durch Messung des Totalionenstromes an den Monitorblenden.

\section{Ergebnisse}

Das durch Trennung an einer gepackten Säule erhaltene Gaschromatogramm (Abb. 1) der Wachse läßt bereits erkennen, daß es sich nicht um eine einfache homologe Reihe von Esterwachsen handelt.

Die nach der Umesterung erhaltenen Fettsäuremethylester zeigen im Ionenstromchromatogramm (Abb. 2) über 40 verschiedene Komponenten, von denen 31 (Peak 2-32, Peak 1=Lösungsmittel) in ihrer Struktur aufgeklärt wurden.

22 S. Meyerson u. L. C. Leitch, J. Amer. chem. Soc. 88, 56 [1966].

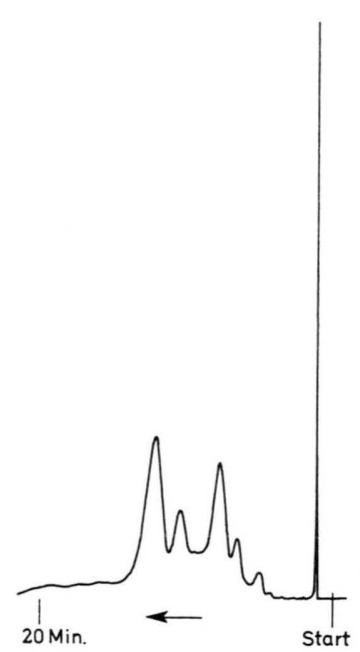

Abb. 1. Gaschromatogramm der Bürzelwachse der Reiherente (2-m-Stahlsäule, stationäre Phase HIEFF/8BP $3 \%$ auf GasChrom Q, $\varnothing 3 \mathrm{~mm}$, Säulentemperatur $250^{\circ}$, Injektionstemperatur $280^{\circ}$, Trägergasströmung $35 \mathrm{ml} \mathrm{N}_{2} / \mathrm{Min}$.).

Alle Komponenten sind methylverzweigte Fettsäuremethylester und zeigen die für diese Stoffklasse charakteristischen Fragmentierungen ${ }^{17,22,23}$,

$$
\left.R-\left(\mathrm{CH}_{2}\right)_{n}\left\{\begin{array}{l}
\mathrm{H} \\
\mathrm{CH}- \\
1 \\
\mathrm{CH}_{3}
\end{array}\right\}\left(\mathrm{CH}_{2}\right)_{m}-\stackrel{\mathrm{COOOCH}}{3}\right)^{\oplus}
$$

die die folgenden Bruchstücke liefern:

$M^{\oplus}, \quad M \cdot \dot{\mathrm{O}} \mathrm{CH}_{3}$ entsprechend $m / e=M-31, \quad M$ $\left(\dot{\mathrm{C}} \mathrm{H}_{2}\right)_{n} \mathrm{R}, M-\left[\left(\underset{\mathrm{C}_{2}}{\mathrm{C}_{2}}\right)_{n} \mathrm{R}+\mathrm{CH}_{3} \mathrm{OH}\right], M-\left[\left(\dot{\mathrm{C}}_{2}\right)_{n} \mathrm{R}+\right.$ $\left.\mathrm{CH}_{3} \mathrm{OH}+\mathrm{H}_{2} \mathrm{O}\right],\left(\stackrel{\oplus}{\mathrm{C}} \mathrm{H}_{2}\right)_{\mathrm{m}} \mathrm{COOCH}_{3}$ entsprechend $\mathrm{m} / \mathrm{e}$ $87,101,115,129 \ldots$ für $m=2,3,4,5 \ldots$ und $\left[\left(\mathrm{CH}_{2}\right)_{m} \mathrm{COOH}_{3}+\mathrm{H},+2 \mathrm{H}\right]^{\oplus}$.

Daneben findet man das durch McLafferty-Umlagerung unter Wanderung des $\gamma-\mathrm{H}$-Atoms entstandene Ion:

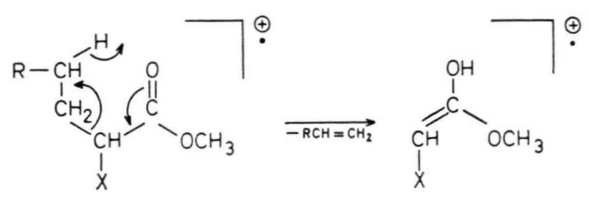

mit $m / e=74$ für $\mathrm{X}=\mathrm{H}$ bzw. $m / e=88$ für $\mathrm{X}=\mathrm{CH}_{3}$.

Diese Umlagerung ermöglicht Substituenten am C-2-Atom sicher zu erkennen.

23 A. Zeman u. H. Scharmann, Fette Seifen einschl. Anstrichmittel, im Druck. 


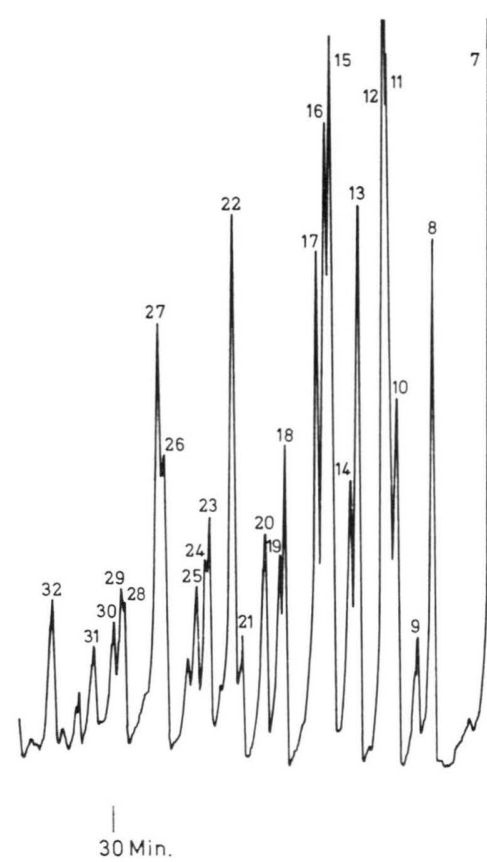

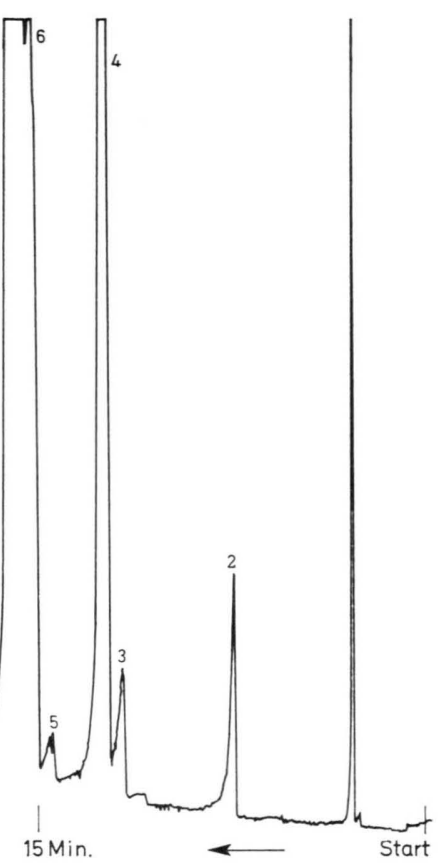

Abb. 2. Ionenstromchromatogramm der Fettsäuremethylester aus dem Bürzelwachs der Reiherente (Bedingungen der GLC s. dort).
Eine weitere Umlagerung unter Eliminierung von (2) $\mathrm{CH}_{3} \mathrm{OH}+\mathrm{CH}_{2}=\mathrm{CHOH}(M-76)$ resp. $\mathrm{CH}_{3} \mathrm{OH}$.<smiles>CC=CC(C)C</smiles>

$(M-90)$

findet nur bei Substitution in 6-Stellung statt und ist zum Erkennen von in 6- oder 2.6-Position substituierten Verbindungen nützlich:
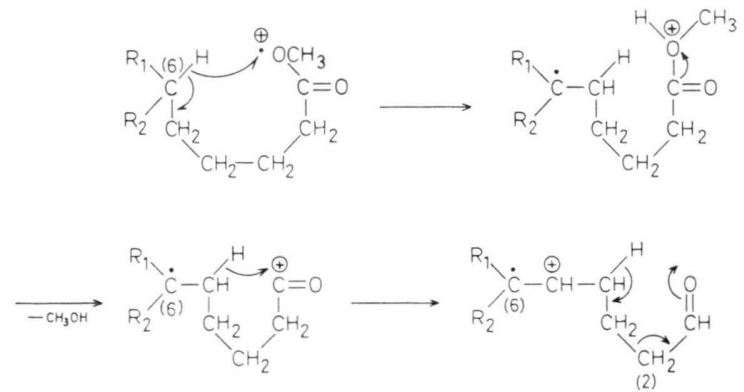

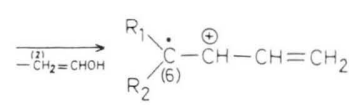

(M-76 bzw. $M-90$ bei Methylsubstitution am C-2-Atom)

Einige zur Identifizierung unbekannter verzweigter Fettsäuremethylester nützliche, empirisch gefundene Regeln wurden von KarLsSON und ODHAM zusammengestellt ${ }^{17}$.
Im folgenden sind die Massenspektren der gaschromatographisch getrennten Methylester (Peak 2 bis 32) diskutiert.

\section{Peak 2}

$M^{\oplus}=72$, ein intensives $\mathrm{McL}$ afferty-Ion $m / e=88$, zusammen mit den intensiven Ionen $m / e=101$ und 129 sowie dem Fehlen von $m / e$ $=115$ deuten auf das Vorliegen von 2.4-Dimethylheptansäuremethylester. Dies wird durch das intensive Ion $M-59\left(M-\dot{\mathrm{COOCH}}{ }_{3}\right)$ bei $m / e=113$ bestätigt.

\section{Peak 3}

$M^{\oplus}=186$, neben der Abspaltung von $\dot{\mathrm{C}} \mathrm{H}_{3}$ $(m / e=171)$ beobachtet man ein intensives Ion $M$ $\dot{\mathrm{C}}_{2} \mathrm{H}_{5}$ mit $m / e=157$, das in einer Folgereaktion $\mathrm{CH}_{3} \mathrm{OH}$ verliert $(m / e=125)$. Das $\mathrm{McLaf}$. ferty-Ion $m / e=88$, intensive Ionen der Serie ${ }^{\oplus}\left(\mathrm{CH}_{2}\right)_{m}-\underset{\mathrm{CH}_{3}}{\mathrm{CH}}-\mathrm{COOCH}_{3}$ mit $m=1 \quad(m / e=101)$, $2(m / e=115), 3(\mathrm{~m} / \mathrm{e}=129)$ und $5(m / e=157)$ identifizieren die Verbindung als 2.6-Dimethyloctansäuremethylester.

Das für 6-Substitution typische Umlagerungs-Ion $M \cdot 76$ (bzw. $M-90)$ d. h. $M \cdot\left(\mathrm{CH}_{3} \mathrm{OH}+\mathrm{CH}_{2}=\right.$ 
CHOH) bzw $\quad M \cdot\left[\mathrm{CH}_{3} \mathrm{OH}+\underset{\mathrm{CH}_{3}}{\mathrm{CH}}=\mathrm{CHOH}\right] \quad$ (s.

l. c. $\left.{ }^{22}\right)$ tritt nur wenig intensiv auf $(m / e=96)$. Seine Bildung ist offenbar abhängig von der Länge der Fettsäure-Kette (vgl. z. B. Massenspektren von Peak 8 und 13). Das nach KarLsson und Odham ${ }^{17}$ zu erwartende Ion $M-49$ wird bei $m / e=137$ beobachtet.

\section{Peak 4}

$M^{\oplus}=200 ;$ im oberen Massenbereich treten die Ionen $M-47 \quad(m / e=153)$ und $M-49 \quad(m / e=151)$ auf. Die Ionen mit $m / e=88,101$ und 129 werden mit großer Intensität gebildet, $m / e=115$ dagegen fehlt, d. h. die zweite Methylgruppe befindet sich am C-4-Atom. Die Eliminierung von $\mathrm{CH}_{3} \mathrm{OH}$ und anschließend von $\mathrm{H}_{2} \mathrm{O}$ aus dem Esterion $m / e=171$ zeigt, da $\beta$ sich in Position 6 eine dritte $\mathrm{CH}_{3}$-Gruppe befindet. Das Massenspektrum ist identisch mit dem von ODHAM ${ }^{11}$ publizierten Spektrum des aus dem Bürzelfett des Schwanes isolierten 2.4.6-Trimethyloctansäuremethylesters.

\section{Peak 5}

$M^{\oplus}=200$, die Ionen $m / e=141(M-59), m / e=88$ und das intensive Ion $m / e=101$ sowie 129 identifizieren diese Komponente als 2.4-Dimethylnonansäuremethylester.

\section{Peak 6}

Die von Peak 7 nur unvollkommen getrennte Komponente hat ein $M^{\oplus}$ bei $m / e=200$. Die intensiven Ionen bei $m / e=88,101,115,129$ und 157 deuten auf das Vorliegen von Methylverzweigungen am C-2- und C-6-Atom. Damit in Übereinstimmung steht das Auftreten der Fragmente 157- $\mathrm{CH}_{3} \mathrm{OH}$ $(m / e=125), M-47, M-49$ und $M-90 \quad(m / e=110)$. Letzteres Ion entsteht durch Eliminierung von $\mathrm{CH}_{3} \mathrm{OH}+\underset{\mathrm{CH}_{3}}{\stackrel{(2)}{\mathrm{C}}}=\mathrm{CHOH} \quad$ (s. l. c. $\left.{ }^{22}\right)$. Die Kompo-

nente Peak 6 ist somit ein Homologes von Peak 3, nämlich 2.6-Dimethylnonansäuremethylester.

\section{Peak 7}

$M^{\oplus}=214$, die Ionen $m / e=88,101,129,143$ und 171 (letzteres spaltet $\mathrm{CH}_{3} \mathrm{OH}$ und $\mathrm{H}_{2} \mathrm{O}$ ab $\rightarrow m / e 139$ und 121$)$ sowie die Ionen $M-49(m / e=$
165) und $M-87(m / e=127)$ identifizieren die Komponente Peak 7 als 2.4.6-Trimethylnonansäuremethylester. Das Massenspektrum ist mit dem der von ODHAM ${ }^{11}$ aus dem Bürzeldrüsensekret des Schwanes isolierten Verbindung identisch.

\section{Peak 8}

$M^{\oplus}=214$, die Ionen $M-49(m / e=165), M-90$ $(m / e=124)$ und das Fehlen des Ester-Ions $m / e=$ 143 zeigen, daß es sich um ein Homologes von Peak 6, um 2.6-Dimethyldecansäuremethylester handelt.

\section{Peak 9}

$M^{\odot}=214$, das McLafferty-Ion tritt bei $m / e=88$ auf und beweist eine Methylsubstitution am C-2-Atom. Eine zweite Methylgruppe befindet sich am C-8-Atom, was aus dem Auftreten der Ionen $M-\dot{\mathrm{C}}_{2} \mathrm{H}_{5} \quad(m / e=185), M-\left(\dot{\mathrm{C}}_{2} \mathrm{H}_{5}+\mathrm{CH}_{3} \mathrm{OH}\right) \quad(m / e=$ 153) und $\mathrm{M}-\left(\dot{\mathrm{C}}_{2} \mathrm{H}_{5}+\mathrm{CH}_{3} \mathrm{OH}+\mathrm{H}_{2} \mathrm{O}\right)$ mit $\mathrm{m} / \mathrm{e}=$ 135 folgt. Die Komponente muß als 2.8-Dimethyldecansäuremethylester gedeutet werden.

Peak 10

$M^{\oplus}=228$. Die gaschromatographische Retentionszeit läßt vermuten, $\mathrm{da} ß$ ein trimethylsubstituierter Methylester vorliegt. Das $\mathrm{McL}$ a f f e r ty Ion $m / e=88$ und das gegenüber $m / e=115 \mathrm{sehr}$ intensive Ion $m / e=129$ (aus $129-32 \rightarrow 97$ ) zeigen Methylsubstitutionen am C-2- und C-4-Atom an. Das Ion $m / e=157$ entspricht dem intensiven Ion $m / e=143$ in den Spektren der $n$-Fettsäuremethylester, das durch $\alpha$-Methylsubstitution um 14 Masseneinheiten verschoben wird. Irrelevant ist auch die große Intensität des Ions $M-43(m / e=185)$, da dieses weitgehend durch Eliminierung der C-Atome 2 und 3 gebildet wird ${ }^{24}$. Die Position der dritten Methylgruppe am C-8-Atom erhält man aus dem Ion $m / e=199$ und seinen Folge-Ionen $m / e=167$ $\left(-\mathrm{CH}_{3} \mathrm{OH}\right)$ und $149\left(-\mathrm{CH}_{3} \mathrm{OH}-\mathrm{H}_{2} \mathrm{O}\right)$. Aus den Spektren folgt, daß es sich um einen 2.4.8-Trimethyldecansäuremethylester handelt (vgl. Abb. 3) .

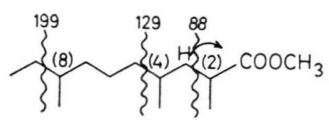

24 I. M. Campbell u. J. Naworal, J. Lipid Res. 10, 593 [1969]. 


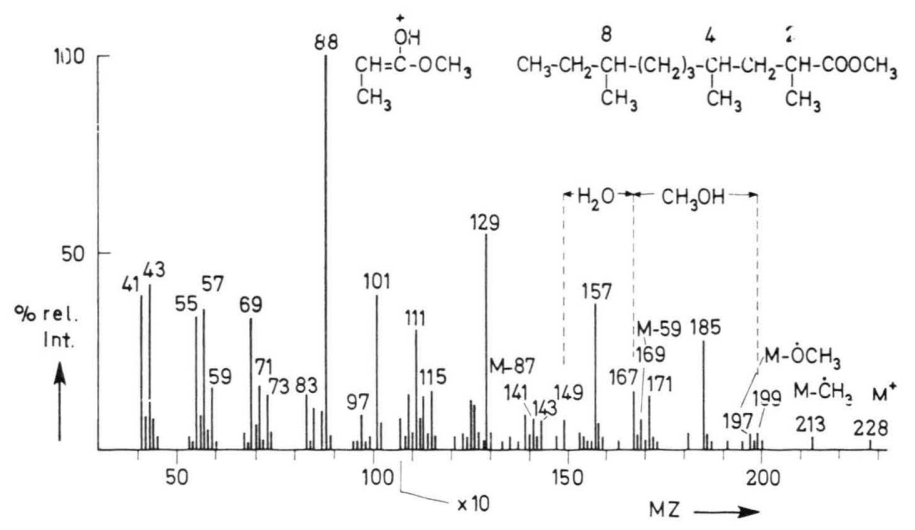

Abb. 3. Massenspektrum der Komponente Peak 10.

Peak 11

$M^{\oplus}=228$. Nach der GLC-Retentionszeit handelt es sich um einen trimethyl-substituierten Fettsäuremethylester. Das McLafferty-Ion tritt bei $m / e=88$ auf und beweist damit eine Methylgruppe am C-2-Atom. Weitere Methylgruppen befinden sich am C-6- und C-8-Atom. Die dafür charakteristischen Ionen werden bei $m / e=138(M-90)$ und 199 $\left(M-\dot{\mathrm{C}}_{2} \mathrm{H}_{5}\right)$ beobachtet, gefolgt von $\mathrm{CH}_{3} \mathrm{OH}$ - und $\mathrm{H}_{2} \mathrm{O}$-Eliminierung $(m / e=167$ und 149$)$.

Das Spektrum ist identisch mit dem des 2.6.8-Trimethyldecansäuremethylesters der aus dem Bürzelwachs der Eiderente isoliert wurde ${ }^{\mathbf{1 4}}$.

Peak 13

$M^{\oplus}=228$. Die charakteristischen Ester-Ionen $m / e=88,101,115,129,157$ und das UmlagerungsIon $138(M-90)$ identifizieren die Komponente als 2.6-Dimethylundecansäuremethylester.

Peak 14

$M^{\oplus}=228$. Hier liegt nach der GLC-Retentionszeit ebenfalls ein dimethylverzweigter Fettsäuremethylester vor. Die Ionen der Ester-Serie werden bei $m / e=88,101,115,129,143,157$ und 185 mit großer Intensität beobachtet. Letzteres Ion eliminiert in einer Folgereaktion $\mathrm{CH}_{3} \mathrm{OH}(m / e=153)$ und $\mathrm{H}_{2} \mathrm{O}$ $(m / e=135)$. Ein Ion $M-59$ tritt bei $m / e=169$ auf. Aus diesen Spaltungen ist zu schließen, daß ein 2.8-Dimethylundecansäuremethylester vorliegt. Dies stimmt mit der von ODHAM ${ }^{14}$ angegebenen Reihenfolge der gaschromatographischen Retentionszeiten für 2.4-, 2.6-, 2.8- und 2.10-disubstituierte Fettsäuremethylester überein.

\section{Peak 15}

$M^{\oplus}=242$. Die Retentionszeit gibt einen Hinweis auf einen trisubstituierten Methylester. Charakteristisch für Methylgruppen am C-2- und C-4-Atom sind die Ionen $m / e=88,101$ und 129 (groß) gegenüber 115 (klein). Die Ionen $m / e=199$ und 167/149 (s. Peak 10) sind mit einer Substitution am C-8-Atom vereinbar. Das in Abb. 3 beobachtete Ion $M-87$ ist hier zu $m / e=155$ verschoben. Die auftretenden Fragmentierungen identifizieren die Komponente 15 als ein Homologes von Peak 10, als 2.4.8-Trimethylundecansäuremethylester.

Peak 16

$M^{\oplus}=242$. Wie bei Peak $15 \mathrm{mu}$ es sich nach der GLC-Retentionszeit um einen trimethylsubstituierten Fettsäuremethylester handeln. Die Intensität der Ester-Ionen $m / e=88,101,115,129,143$ (sehr klein), 157, 171, 185 (sehr klein) und 199 deutet auf Methylgruppen am C-2-, C-6- und C-8-Atom. Dies wird durch die Ionen $m / e=167\left(M-\left[\dot{\mathrm{C}}_{2} \mathrm{H}_{5}+\right.\right.$ $\left.\left.\mathrm{CH}_{3} \mathrm{OH}\right]\right), \quad m / e=149 \quad\left(M-\left[\dot{\mathrm{C}}_{2} \mathrm{H}_{5}+\mathrm{CH}_{3} \mathrm{OH}+\right.\right.$ $\left.\mathrm{H}_{2} \mathrm{O}\right]$ ) und $m / e=152(M-90)$ bestätigt. Die Komponente Peak 16 ist identisch mit 2.6.8-Trimethylundecansäuremethylester und damit homolog zu Peak 11.

\section{Peak 17}

$M^{\odot}=228$. Das Mc-Laffery-Ion wird bei $m / e=88$ beobachtet. Anhand der Intensitäten der Ester-Ionen $\left(\stackrel{\oplus}{\mathrm{C}} \mathrm{H}_{2}\right)_{\mathrm{m}} \mathrm{COOCH}_{3}$ mit $m / e=101,115 \ldots$ und einem intensiven Ion $m / e=169(M-59$ bzw. $M-\mathrm{COOCH}_{3}$ ) läßt sich diese Komponente als 2-Methyldodecansäuremethylester identifizieren. 
Peak 18

$M^{\oplus}=242$. Die GLC-Retentionszeit läßt vermuten, daß ein dimethyl-substituierter Fettsäuremethylester vorliegt. Ein sehr intensives Ion $M-90(m / e=152)$ zusammen mit dem McLafferty - Ion $m / e=88$ beweisen das Vorliegen von 2.6-Dimethyldodecansäuremethylester. Die gegenüber dem Ester-Ion mit $m / e=115$ besonders große Intensität des homologen Ions $m / e=129$ sowie das Ion $M-59$ zeigen, daß im Peak 18 neben dem 2.6-Isomeren wahrscheinlich auch das 2.4-Isomere enthalten ist.

Peak 19

$M^{\oplus}=242$. Auch hier sollte nach der GLC-Retentionszeit ein disubstituierter Ester vorliegen. Die Ionen $m / e=88$, und $185 \quad\left(185-\mathrm{CH}_{3} \mathrm{OH}=153\right.$; $153-\mathrm{H}_{2} \mathrm{O}=135$ ) identifizieren Komponente Peak 19 als ein Homologes von Peak 14, als 2.8-Dimethyldodecansäuremethylester.

\section{Peak 20}

$M^{\oplus}=242$. Das Ion $m / e=88$ und die $\mathrm{CH}_{3} \mathrm{OH}$ und $\mathrm{H}_{2} \mathrm{O}$-Eliminierung aus dem Ester-Ion $m / e=213$ $\left(M-\dot{\mathrm{C}}_{2} \mathrm{H}_{5}\right) \quad\left(213-\mathrm{CH}_{3} \mathrm{OH}=181 ; 181-\mathrm{H}_{2} \mathrm{O}=\right.$ 163) beweisen, daß es sich um 2.10-Dimethyldodecansäuremethylester handelt. Diese Zuordnung wird durch die GLC-Retentionszeit bestätigt.

Peak 21

$M^{\oplus}=242$. Das Ion $M-59 \quad(m / e=183)$, das McLafferty-Ion $m / e=88$ und die Intensitäten in der Ester-Ionen-Serie<smiles>CCC(C)C(=O)OC</smiles>

sprechen für einen am C-2-Atom monomethylsubstituierten Tridecansäuremethylester. Dieses Ergebnis stimmt mit den GLC-Befunden überein.

\section{Peak 22}

$M^{\oplus}=256$. Die für 2- und 6-Substitution typischen Ionen treten bei $m / e=88$ und $166(M-90)$ auf (vgl. Abb. 4).

Die Position einer dritten Methylgruppe am C-10-Atom läßt sich mittels der Ionen $m / e=227$, $195\left(=227-\mathrm{CH}_{3} \mathrm{OH}\right) \quad$ und $177 \quad(=227$ $-\mathrm{CH}_{3} \mathrm{OH}-\mathrm{H}_{2} \mathrm{O}$ ) ableiten. Ionen $M-49$ und $M-47$ werden bei $m / e=207$ und 209 beobachtet. Das Massenspektrum ist mit dem Vorliegen eines 2.6.10Trimethyldodecansäuremethylesters vereinbar

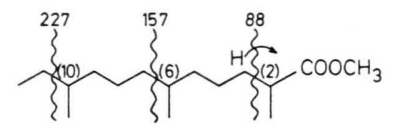

Peak 23/24/25

Alle drei Komponenten haben ein $M^{\oplus}=256$. Dies deutet zusammen mit den GLC-Retentionszeiten darauf hin, daß isomere dimethyl-substituierte Tridecansäuremethylester vorliegen. Im Spektrum der Komponente 23 treten das McLafferty-Ion bei $m / e=88$ und das für 2.6-Substitutionen charakteristische Ion $M-90$ bei $m / e=166$ auf und identifizieren sie als 2.6-Dimethyltridecansäuremethylester.

Die Komponenten 24 und 25 haben ebenfalls Methylgruppen am C-2- und am C-8- bzw. C-10Atom. Die dafür typischen Ester-Ionen mit ihren Folgeionen werden bei $m / e=185$ (mit 185 $-\mathrm{CH}_{3} \mathrm{OH}=153$ und $153-\mathrm{H}_{2} \mathrm{O}=135$ ) und $m / e$

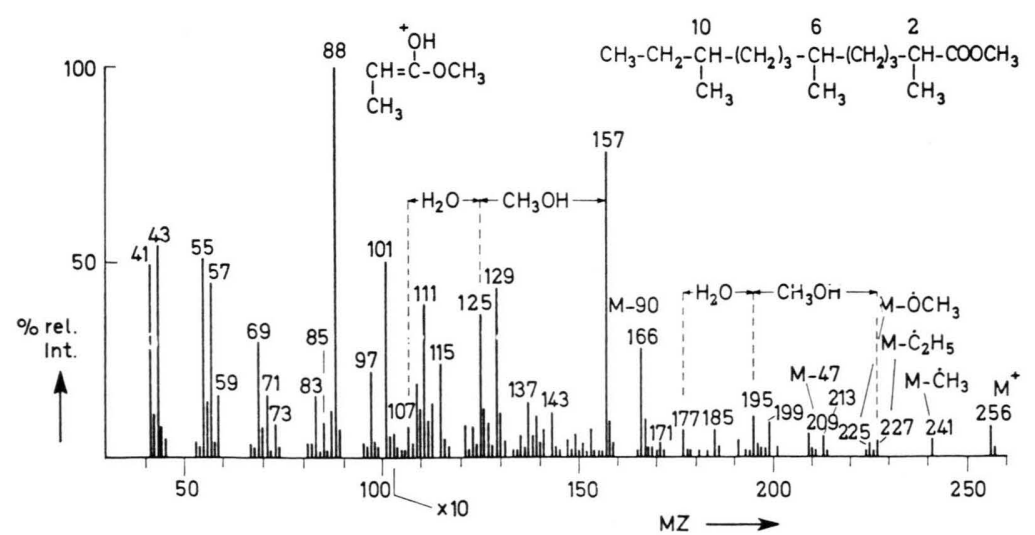

Abb. 4. Massenspektrum der Komponente Peak 22. 
$=213\left(\right.$ mit $213-\mathrm{CH}_{3} \mathrm{OH}=181$ und $181-\mathrm{H}_{2} \mathrm{O}=$ 163) beobachtet.

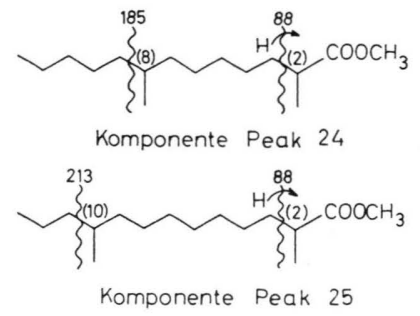

Die Komponenten 24 und 25 sind somit Homologen der Verbindungen der Peaks 19 und 20.

Peak 26

$M^{\oplus}=270$. Die Ionen $m / \mathrm{e}=88$ und $180(M-90)$ beweisen die 2.6-Substitution. Eine weitere Methylgruppe befindet sich am C-10-Atom, da das Ion $m / e=227$ Methanol und $\mathrm{H}_{2} \mathrm{O}$ eliminiert $(m / e=$ 195 und 177). Die Ionen $M-47$ und $M-49$ (vgl. Peak 22) treten bei $m / e=221$ und 223 auf. Die beobachteten Ionen identifizieren die vorliegende Komponente als ein Homologes von Peak 22, nämlich als 2.6.10-Trimethyltridecansäuremethylester.

\section{Peak 27}

$M^{\oplus}=256$. Aus der GLC-Retentionszeit und den Ionen $m / e=88$ und $197(M-59)$ läßt sich ersehen, daß ein 2-Methyltetradecansäuremethylester vorliegt.

\section{Peak 28/29/30}

Die Verbindungen sind gaschromatographisch kaum getrennt; trotzdem ist aus der GLC-Retentionszeit und den Massenspektren der Gemische eine eindeutige Identifizierung möglich. Alle Komponenten haben ein $M^{\oplus}=270$, d. h. es handelt sich, wenn man die Retentionszeit berücksichtigt, um dimethylsubstituierte Tetradecansäuremethylester. Die charakteristischen Ionen sind identisch mit denen in den Massenspektren der Komponenten 23, 24 und 25, d. h. es liegen die Homologen 2.6-, 2.8- und 2.10-Dimethyltetradecansäuremethylester vor.

\section{Peak 31}

$M^{\oplus}=270$, ist ein Homologes von Peak 27 und kann als 2-Methylpentadecansäuremethylester identifiziert werden.

\section{Peak 32}

$M^{\oplus}=284$. Die GLC-Retentionszeit und die Ionen $m / e=88$ und 194 (M-90) identifizieren diese Ver- bindung als 2.6-Dimethyltetradecansäuremethylester.

Die quantitative Zusammensetzung der aus dem Bürzelwachs der Reiherente isolierten Fettsäuren ist in Tab. 1 wiedergegeben.

\begin{tabular}{|c|c|c|}
\hline $\begin{array}{l}\text { Peak-Nr. } \\
\text { in Abb. } 2\end{array}$ & $\begin{array}{c}\text { Struktur der Fettsäure als } \\
\text { Bestandteil des Bürzelwachses }\end{array}$ & $\begin{array}{l}\text { Anteil in } \\
\text { Flächen } \%\end{array}$ \\
\hline 2 & 2.4-Dimethylheptansäure & 0,3 \\
\hline 3 & 2.6-Dimethyloctansäure & 0,5 \\
\hline 4 & 2.4.6-Trimethyloctansäure & 8,7 \\
\hline 5 & 2.4-Dimethylnonansäure & 0,2 \\
\hline 6 & 2.6-Dimethylnonansäure & 5,9 \\
\hline 7 & 2.4.6-Trimethylnonansäure & 39,0 \\
\hline 8 & 2.6-Dimethyldecansäure & 2,1 \\
\hline 9 & 2.8-Dimethyldecansäure & 0,7 \\
\hline 10 & 2.4.8-Trimethyldecansäure & 1,5 \\
\hline 11 & 2.6.8-Trimethyldecansäure & 2,6 \\
\hline 12 & 2.4.6.8-Tetramethyldecansäure & 3,6 \\
\hline 13 & 2.6-Dimethylundecansäure & 2,5 \\
\hline 14 & 2.8-Dimethylundecansäure & 1,3 \\
\hline 15 & 2.4.8-Trimethylundecansäure & 3,5 \\
\hline 16 & 2.6.8-Trimethylundecansäure & 2,7 \\
\hline 17 & 2-Methyldodecansäure & 2,5 \\
\hline 18 & 2.4-Dimethyldodecansäure und $\}$ & 1,5 \\
\hline 19 & 2.8-Dimethyldodecansäure & 1,0 \\
\hline 20 & 2.10-Dimethyldodecansäure & 1,6 \\
\hline 21 & 2-Methyltridecansäure & 0,7 \\
\hline 22 & 2.6.10-Trimethyldodecansäure & 3,3 \\
\hline 23 & 2.6-Dimethyltridecansäure & 20 \\
\hline 24 & 2.8-Dimethyltridecansäure & 2,0 \\
\hline 25 & 2.10-Dimethyltridecansäure & 0,9 \\
\hline 26 & 2.6.10-Trimethyltridecansäure & 1,6 \\
\hline 27 & 2-Methyltetradecansäure & 2,4 \\
\hline 28 & 2.6-Dimethyltetradecansäure & 1,2 \\
\hline 29 & 2.8-Dimethyltetradecansäure & 1,2 \\
\hline 30 & 2.10-Dimethyltetradecansäure & 0,7 \\
\hline 31 & 2-Methylpentadecansäure & 0,7 \\
\hline 32 & 2.6-Dimethylpentadecansäure & 1,0 \\
\hline- & nicht identifiziert & 3,8 \\
\hline
\end{tabular}

Tab. 1. Quantitative Zusammensetzung der aus dem Bürzelwachs der Reiherente isolierten Fettsäuren (in Flächen-\%).

Im Ionenstromchromatogramm (Abb. 5) der durch Chromsäureoxidation aus den Alkoholen erhaltenen Fettsäuremethylester treten ca. 30 Komponenten auf.

Von 16 Komponenten wurden Massenspektren erhalten. Die Komponenten der Peaks 1, 2, 4, 7, 12 und 14 ließen sich mit Hilfe der gaschromatographischen Retentionszeiten und der massenspektrometrischen Befunde als $n-\mathrm{C}_{12^{-}}, \mathrm{C}_{13^{-}}, \mathrm{C}_{14^{-}}, \mathrm{C}_{16^{-}}, \mathrm{C}_{17^{-}}$ und $\mathrm{C}_{18}$-Methylester identifizieren.

Von besonderem Interesse sind die Bereiche der methylverzweigten Methylester mit Kettenlängen von 17 und 19 C-Atomen. Die gute Trennleistung der GLC-MS-Kombination erlaubte diese bei ande- 


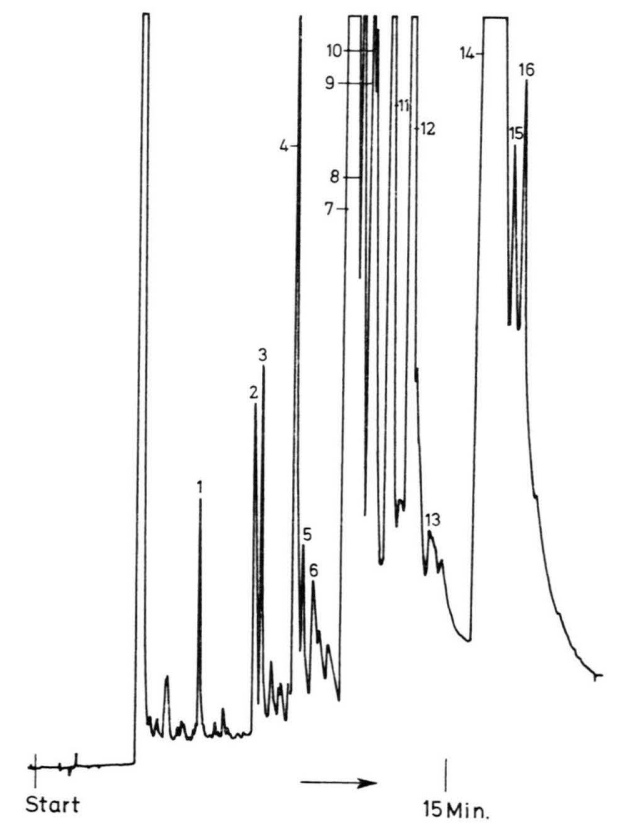

Abb. 5. Ionenstromchromatogramm der aus den Wachsalkoholen resultierenden Fettsäuremethylester.

ren Bürzelwachskomponenten bislang nur unvollkommen getrennten Bereiche genauer zu untersuchen.

Im Bereich der verzweigten Methylester mit 17 C-Atomen ließen sich folgende Verbindungen identifizieren.

\section{Peak 9}

$M^{\oplus}=284$. Nach der GLC liegt ein mono-methylsubstituierter Fettsäuremethylester vor. Bei verzweigten Fettsäuremethylestern mit längerer Alkylkette treten durch Fragmentierung an der Verzweigungsstelle charakteristische Ester-Ionen und ihre um $\mathrm{H}$ und $2 \mathrm{H}$ reicheren Begleiter auf. Diese Ionen werden bei $m / e=171,172$ und $173\left[\left(\mathrm{CH}_{2}\right)_{8} \mathrm{COOCH}_{3}\right.$, $+\mathrm{H},+2 \mathrm{H}]^{\oplus}$ mit hoher Intensität beobachtet. Sie weisen auf eine Substitution am C-10-Atom hin. Das bei 10-Substitution zu erwartende Fragment $m / e=$ 199 tritt intensiv auf und verliert erwartungsgemäß $\mathrm{CH}_{3} \mathrm{OH}(m / e=167)$ und $\mathrm{H}_{2} \mathrm{O}(m / e=149)$.

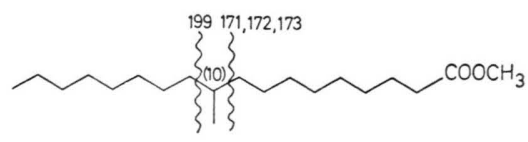

Eine detaillierte Interpretation des Massenspektrums zeigt, daß neben 10-Methylhexadecansäuremethylester in geringerer Konzentration noch wei- tere Isomere in Peak 9 enthalten sein müssen. Den Ionen-Serien

$\stackrel{\stackrel{\oplus}{\mathrm{C}} \mathrm{H}-\left(\mathrm{CH}_{2}\right)_{n}-\mathrm{COOCH}_{3}-\left(\mathrm{CH}_{3} \mathrm{OH}\right) \text { und }}{\mathrm{CH}_{3}} \stackrel{\stackrel{\oplus}{\mathrm{C}} \mathrm{H}-\left(\mathrm{CH}_{2}\right)_{n}-\mathrm{COOCH}_{3}-\left(\mathrm{CH}_{3} \mathrm{OH}+\mathrm{H}_{2} \mathrm{O}\right)}{\mathrm{CH}_{3}}$

zugehörige Ionen treten bei $m / e=107,125$ (7-Substitution), 121, 139 (8-Substitution) und 135, 153 (9-Substitution) auf.

Peak 10

$M^{\oplus}=284$. Für die Erkennung der Verzweigungsstelle wichtige Ionen treten bei $m / e=199,200,201$ $\left[\left(\mathrm{CH}_{2}\right)_{10} \mathrm{COOCH}_{3},+\mathrm{H},+2 \mathrm{H}\right]^{\oplus}$ auf, sowie bei $m / e=227 \quad$ (daneben $195=227-\mathrm{CH}_{3} \mathrm{OH}$ und $\left.177=195-\mathrm{H}_{2} \mathrm{O}\right)$. Die Hauptkomponente in Peak 10 stellt somit 12-Methylhexadecansäuremethylester dar. In geringer Menge sind Isomeren mit Methylverzweigungen am C-7-, C-8-, C-9- und C-10-Atom nachweisbar. $(m / e=107 / 125 ; 121 / 139$; $135 / 153$ und $149 / 167)$.

\section{Peak 11}

$M^{\oplus}=284$. Die für einen anteiso-Ester charakteristischen Ionen $M \cdot \dot{\mathrm{C}}_{2} \mathrm{H}_{5}, M-\left(\dot{\mathrm{C}}_{2} \mathrm{H}_{5}+\mathrm{CH}_{3} \mathrm{OH}\right)$ und $M-\left(\dot{\mathrm{C}}_{2} \mathrm{H}_{5}+\mathrm{CH}_{3} \mathrm{OH}+\mathrm{H}_{2} \mathrm{O}\right)$ treten bei $m / e=255$, 223 und 205 auf. Die durch Spaltung an der Verzweigungsstelle gebildeten Esterfragmente werden bei $m / e=227,228$ und 229 beobachtet.

Bei der Komponente 11 handelt es sich daher um 14-Methylhexadecansäuremethylester.

Im Bereich der verzweigten Methylester mit 19 C-Atomen wurden Peak 15 und 16 identifiziert.

\section{Peak 15}

$M^{\oplus}=312$. Die GLC-Retentionszeit deutet auf einen mittelständig monomethyl-substituierten Fettsäuremethylester. Aus den Ionen $m / e=236$ (M-76, d.i. $\left.\mathrm{CH}_{3} \mathrm{OH}+\mathrm{CH}_{2}=\mathrm{CHOH}\right), 171,172$ und 173, d.i. $\left[\left(\mathrm{CH}_{2}\right)_{8} \mathrm{COOCH}_{3},+\mathrm{H},+2 \mathrm{H}\right]^{\oplus}$ sowie $199 \mathrm{mit}$ den resultierenden Ionen $167=199-\mathrm{CH}_{3} \mathrm{OH}$ und $149=167-\mathrm{H}_{2} \mathrm{O}$ läßt sich ersehen, daß ein Gemisch aus 6- und 10-Methyloctadecansäuremethylester vorliegt. Die intensiven Ionen $m / e=139$ und $121\left[171-\mathrm{CH}_{3} \mathrm{OH}\right.$ bzw. $\left.171-\left(\mathrm{CH}_{3} \mathrm{OH}+\mathrm{H}_{2} \mathrm{O}\right)\right]$ lassen vermuten, $\mathrm{da} ß \mathrm{im}$ Gemisch in geringer Menge auch das 8-Isomere enthalten ist. 
Peak 16

$M^{\oplus}=312$. Die Hauptkomponente in Peak 16 wird durch die Ester-Ionen $m / e=227,228,229$ $\left[\left(\mathrm{CH}_{2}\right)_{12} \mathrm{COOCH}_{3},+\mathrm{H},+2 \mathrm{H}\right]$ und durch $M$ $\left(\dot{\mathrm{C}}_{4} \mathrm{H}_{9}+\mathrm{CH}_{3} \mathrm{OH}+\mathrm{H}_{2} \mathrm{O}\right)$ mit $m / e=205$ sowie durch $m / e=255 \quad\left(M-\dot{\mathrm{C}}_{4} \mathrm{H}_{9}\right) \quad$ und $223 \quad\left[M-\left(\dot{\mathrm{C}}_{4} \mathrm{H}_{9}+\right.\right.$ $\left.\mathrm{CH}_{3} \mathrm{OH}\right)$ ] als 14-Methyloctadecansäuremethylester identifiziert. Daneben werden gemäß der entsprechenden Ionen-Serien die Isomeren mit der Methylgruppe am C-7-, C-8-, C-9-, C-10-, C-11- und C-12Atom nachgewiesen.

Die gaschromatographisch ermittelte quantitative Zusammensetzung der aus den Alkoholen durch Chromsäure-Oxidation erhaltenen Fettsäuremethylester gibt für die ersteren das in Tab. 2 wiedergegebene Bild.

\begin{tabular}{clc}
\hline $\begin{array}{c}\text { Peak Nr. } \\
\text { in Abb. } 5\end{array}$ & \multicolumn{1}{c}{ Struktur des Alkohols } & $\begin{array}{r}\text { Anteil in } \\
\text { Flächen- } \%\end{array}$ \\
\hline 1 & $n$-Dodecanol & 0,4 \\
2 & $n$-Tridecanol & 0,6 \\
3 & 2-Methyl-tridecanol & 0,7 \\
4 & n-Tetradecanol & 2,0 \\
5 & 2-Methyl-pentadecanol & 0,5 \\
6 & nicht identifiziert & 0,7 \\
7 & n-Hexadecanol & 22,4 \\
8 & 2-Methyl-hexadecanol & 3,2 \\
9 & 10-Methyl-hexadecanol & 3,3 \\
10 & 12-Methyl-hexadecanol & 2,1 \\
11 & 14-Methyl-hexadecanol & 5,6 \\
12 & $n$-Heptadecanol & 5,8 \\
13 & 2- sowie 10-Methyl-heptadecanol & 0,3 \\
14 & n-Octadecanol & 41,5 \\
15 & 6- sowie 10-Methyl-octadecanol & 2,9 \\
16 & 14-Methyl-octadecanol & 4,6 \\
- & nicht identifiziert & 3,4 \\
\hline
\end{tabular}

Tab. 2. Gaschromatographisch ermittelte Zusammensetzung der Wachsalkohole (aus den Oxydationsprodukten bestimmt).

\section{Diskussion}

Im Bürzelwachs der Reiherente (Aythya fuligula) treten als saure Komponenten mono-(2-Methyl-), di-(2.4-, 2.6-, 2.8- und 2.10-Dimethyl-), tri-(2.4.6-, 2.4.8-, 2.6.8- und 2.6.10-Trimethyl-) und tetramethylsubstituierte Fettsäuren mit Kettenlängen

25 Ng. Dinh-NguyêN, R. Ryhage, S. Ställberg-Stenhagen u. E. Stenhagen, Ark. Kemi 18, 393 [1961].

26 R. Noble, R. Stjerholm, D. Mercier u. E. Lederer, Nature [London] 199, 600 [1963].

27 H. Grisebach u. Achenbach, Z. Naturforsch. 17 b, 634 [1962].

28 A. J. Birch, E. Pride, R. W. Richards u. P. J. Thomson, Chem. and Ind. 1960, 1245. von $\mathrm{C}_{7}-\mathrm{C}_{15}$ auf. Unverzweigte Fettsäuren kommen nicht vor.

Als alkoholische Komponenten finden sich unverzweigte primäre Alkanole mit Kettenlängen zwischen $\mathrm{C}_{12}$ und $\mathrm{C}_{18}$ neben verzweigten primären Alkoholen mit Verzweigungen in 2-, 6-, 7-, 8-, 9-, 10-, 11-, 12- und 14-Position. Andere monomethylsubstituierte Alkanole sind als Spurenkomponenten nicht auszuschließen.

Die Biosynthese polymethyl-verzweigter Makrolide sowie einiger Fettsäuren aus Propionyl-CoA erscheint nach den Arbeiten zahlreicher Autoren geklärt ${ }^{26-31}$. Auffällig ist jedoch im vorliegenden Fall sowie bei anderen Vertretern der 17. Ordnung, daß stets eine Methylverzweigung am C-2-Atom vorliegt, wie es z. B. auch bei der kürzlich untersuchten Saatkrähe ${ }^{19}$ aus der 32 . Ordnung beobachtet wurde. Wir deuten diesen Befund wie an anderer Stelle vorgeschlagen ${ }^{19}$ damit, daß der terminale Schritt der Fettsäurebiosynthese - die Ablösung des Endproduktes von der Synthetase - nur mit Methylmalonyl-CoA möglich ist, sofern die Biosynthese überhaupt an einer üblichen Synthetase abläuft. Stütze dieser Hypothese ist das Fehlen von Fettsäuren mit der 1. Methylverzweigung in einer anderen als der 2-Position. Eine einzige Ausnahme scheint in der 17. Ordnung bei der Eiderente vorzuliegen ${ }^{14}$, wo das Vorkommen einer 4.6-Dimethyloctansäure beobachtet wurde.

Der Einbau von Methyl-malonyl-CoA erfolgt in 1. Näherung statistisch, da fast alle in Frage kommenden Positionen auch tatsächlich realisiert werden (z. B. 2.4-, 2.6-, 2.8-, 2.10-, 2.4.6-, 2.4.8-, 2.6.8-, 2.6.10- und 2.4.6.8).

Die bislang ungeklärte Biosynthese der Wachsalkohole verläuft offenbar nach einem anderen Mechanismus, was sich an den Verzweigungspositionen erkennen läßt, von denen die 2-, 6-, 7-, 8-, 9-, 10-, 11-, 12- und 14-Position beobachtet wurden. Hier erscheint ein Methylübertragungs-Mechanismus wahrscheinlicher.

29 H. Grisebach u. W. Hofheinz, J. Roy. Inst. Chem. 1964, 332.

30 T. Kaneda, C. Butte, S. B. Taubman u. J. W. Corcoran, J. biol. Chemistry 237, 322 [1962].

31 M. Berry, Quart. Rev. (chem. Soc. London) 17, 343 [1963]. 
Stellt man die bisherigen Ergebnisse aus den Untersuchungen der 17. Ordnung einander gegenüber, so ergibt sich:

Alle Arten zeigen in ihren Bürzellipiden das Auftreten von methylverzweigten Fettsäuren, deren

\begin{tabular}{|c|c|}
\hline $\begin{array}{l}\text { Im Bürzelwachs vor- } \\
\text { kommender Fettsäure- } \\
\text { Typ }\end{array}$ & Untersuchte Art \\
\hline tetramethyl-verzweigt & Anser anser (Graugans) ${ }^{9}$ \\
\hline \multirow[t]{2}{*}{$\begin{array}{l}\text { tetra- und trimethyl- } \\
\text { verzweigt }\end{array}$} & $\begin{array}{l}\text { Dendrocygna viduata (Witwenente) } \\
14\end{array}$ \\
\hline & $\begin{array}{l}\text { Cygnus olor (Höckerschwan) } \\
\text { Branta leucopsis (Weißwangen- } \\
\text { gans) } \\
\text { Tadorna tadorna (Brandgans) } \\
\text { Tadorna ferruginea (Rostgans) }\end{array}$ \\
\hline $\begin{array}{l}\text { tetra-, tri- und di- } \\
\text { methyl-verzweigt }\end{array}$ & Somateria mollissima (Eiderente) ${ }^{14}$ \\
\hline $\begin{array}{l}\text { tetra-, tri-, di- und } \\
\text { mono-methyl-verzweigt }\end{array}$ & Aythya fuligula (Reiherente) \\
\hline monomethyl-verzweigt & $\begin{array}{l}\text { Pekingente } 10 \\
\text { Anas platyrhynchos (Stockente) }{ }^{15}\end{array}$ \\
\hline trimethyl-verzweigt & $\begin{array}{l}\text { Cairina moschata (Moschusente) })^{14} \\
\text { Mergus serrator (Mittelsäger) }\end{array}$ \\
\hline
\end{tabular}

Tab. 3. Fettsäuretyp im Bürzelwachs verschiedener Anatiden.
Verzweigungsgrad in Abhängigkeit von der Art in Tab. 3 wiedergegeben ist.

Nimmt man die Zusammensetzung der einzelnen Wachsalkohole hinzu, so zeigen sich in dieser Ordnung deutliche Verwandtschaften. Von den angeführten Arten kommen unverzweigte Alkohole nur bei Dendrocygna viduata, Anser anser, Cairina moschata, Mergus serrator, Tadorna tadorna und Tadorna ferruginea vor; bei den anderen Arten finden sich daneben wechselnde Mengen an methylverzweigten Alkanolen. Chemotaxonomisch lassen sich Verwandtschaften zwischen Dendrocygna und Tadorna, die aus verschiedenen Unterfamilien stammen, sowie zwischen Cairina und Mergus feststellen, die unterschiedlichen Tribus der gleichen Unterfamilie angehören. Die hier untersuchte Art (Aythya fuligula) gleicht im wesentlichen der Eiderente.

Weitere Untersuchungen werden nötig sein, um biologische Querverbindungen deutlich werden zu lassen.

32 G. Niethammer, K. M. Bauer u. U. N. Glutz v. BlotzHEIM, in: Handbuch der Vögel Europas, Bd. 2, Teil 1. Akadem. Verlagsges. Frankfurt/M. 1968. 